\title{
ON INTERSECTIONS OF CONJUGACY CLASSES AND BRUHAT CELLS
}

\author{
KEI YUEN CHAN, JIANG-HUA LU, SIMON KAI-MING TO
}

\begin{abstract}
For a connected semi-simple algebraic group $G$ over an algebraically closed field $\mathbf{k}$ and a fixed pair $\left(B, B^{-}\right)$of opposite Borel subgroups of $G$, we determine when the intersection of a conjugacy class $C$ in $G$ and a double coset $B w B^{-}$ is nonempty, where $w$ is in the Weyl group $W$ of $G$. The question comes from Poisson geometry, and our answer is in terms of the Bruhat order on $W$ and an involution $m_{C} \in W$ associated to $C$. We prove that the element $m_{C}$ is the unique maximal length element in its conjugacy class in $W$, and we classify all such elements in $W$. For $G=S L(n+1, \mathbf{k})$, we describe $m_{C}$ explicitly for every conjugacy class $C$, and when $w \in W \cong S_{n+1}$ is an involution, we give an explicit answer to when $C \cap(B w B)$ is nonempty.
\end{abstract}

\section{INTRODUCTION}

1.1. The set up and the results. Let $G$ be a connected semi-simple algebraic group over an algebraically closed field $\mathbf{k}$, and let $B$ and $B^{-}$be a pair of opposite Borel subgroups of $G$. Then $H=B \cap B^{-}$is a Cartan subgroup of $G$. Let $W=N_{G}(H) / H$ be the Weyl group, where $N_{G}(H)$ is the normalizer of $H$ in $G$. One then has the well-known Bruhat decompositions

$$
G=\bigsqcup_{w \in W} B w B=\bigsqcup_{w \in W} B w B^{-} \quad \text { (disjoint unions). }
$$

Subsets of $G$ of the form $B w B$ or $B w^{\prime} B^{-}$, where $w, w^{\prime} \in W$, will be called Bruhat cells in $G$. For a subset $X$ of $G$, let $\bar{X}$ be the Zariski closure of $X$ in $G$. The Bruhat order on $W$ is the partial order defined by

$$
w_{1} \leq w_{2} \quad \Longleftrightarrow \quad B w_{1} B \subset \overline{B w_{2} B}, \quad w_{1}, w_{2} \in W .
$$

Given a conjugacy class $C$ of $G$, let

$$
\begin{aligned}
W_{C} & =\{w \in W: C \cap(B w B) \neq \emptyset\}, \\
W_{C}^{-} & =\left\{w \in W: C \cap\left(B w B^{-}\right) \neq \emptyset\right\} .
\end{aligned}
$$

The sets $W_{C}$ have been studied by several authors (see, for example, [10, 11] by E. Ellers and N. Gordeev and 4 by G. Carnovale) and are not easy to determine even for the case of $G=S L(n, \mathbf{k})$ (see [11]). On the other hand, for a conjugacy class $C$ in $G$, let 
$m_{C}$ be the unique element in $W$ such that $C \cap\left(B m_{C} B\right)$ is dense in $C$ (see Lemma 2.4). Our first result, Theorem 2.5 , states that, for every conjugacy class $C$ in $G$,

$$
W_{C}^{-}=\left\{w \in W: w \leq m_{C}\right\} .
$$

Thus the set $W_{C}^{-}$is completely determined by $m_{C}$ and the Bruhat order on $W$.

Theorem 2.5 is motivated by Poisson geometry. It is shown in [13] that when $\mathbf{k}=\mathbb{C}$, the connected complex semi-simple Lie group $G$ carries a holomorphic Poisson structure $\pi_{0}$, invariant under conjugation by elements of $H$, such that the nonempty intersections $C \cap\left(B w B^{-}\right)$are exactly the $H$-orbits of symplectic leaves of $\pi_{0}$, where $C$ is a conjugacy class in $G$ and $w \in W$. To describe the symplectic leaves of $\pi_{0}$, one first needs to determine when $C \cap\left(B w B^{-}\right)$is nonempty. By [21, Theorem 1.4], the nonempty intersections $C \cap\left(B w B^{-}\right)$are always smooth and irreducible. The geometry of such intersections and applications to Poisson geometry will be carried out elsewhere.

The elements $m_{C}$ play an important role in the study of spherical conjugacy classes, i.e., conjugacy classes in $G$ on which the $B$-action by conjugation has a dense orbit. Let $\mathbf{k}=\mathbb{C}$ and let $\mathfrak{g}$ be the Lie algebra of $G$. In connection with their proof of the de ConciniKac-Procesi conjecture on representations of the quantized universal enveloping algebra $\mathcal{U}_{\epsilon}(\mathfrak{g})$ at roots of unity over spherical conjugacy classes, N. Cantarini, G. Carnovale, and M. Costantini proved [2, Theorem 25] that a conjugacy class $C$ in $G$ is spherical if and only if $\operatorname{dim} C=l\left(m_{C}\right)+\operatorname{rank}\left(1-m_{C}\right)$, where $l$ is the length function on $W$, and $\operatorname{rank}\left(1-m_{C}\right)$ is the rank of the operator $1-m_{C}$ on the Lie algebra of $H$ (see also [3] if $\operatorname{char}(\mathbf{k}) \neq 2$ and is good for $G$ ). Moreover, it is shown by M. Costantini [6], again for a spherical conjugacy class $C$, that the decomposition of the coordinate ring of $C$ as a $G$-module is almost entirely determined by the element $m_{C}$ (see [․ Theorem 3.22] for the precise statement). When $G$ is simple, the explicit description of $m_{C}$ for each spherical conjugacy class $C$ in $G$ is given in [2], and the complete list of the $m_{C}$ 's, for $C$ spherical, is given in [3, Corollary 4.2].

In the second part of the paper, we study the element $m_{C}$ for an arbitrary conjugacy class $C$ in $G$. After examining some properties of $W_{C}$, we show in Corollary 2.11 that for each conjugacy class $C$ in $G, m_{C} \in W$ is the unique maximal length element in its conjugacy class in $W$. In particular, $m_{C}$ is an involution. When $C$ is spherical, the fact that $m_{C}$ is an involution is also proved in [2, Remark 4] and [3, Theorem 2.7]. Let

$$
\mathcal{M}=\left\{m \in W: m \text { is the unique maximal length element in } \mathcal{O}_{m}\right\},
$$

where $\mathcal{O}_{m}$ is the conjugacy class of $m$ in $W$. Then $m_{C} \in \mathcal{M}$ for every conjugacy class $C$ in $G$. It is thus desirable to study the set $\mathcal{M}$.

Assume that $G$ is simple. Using arguments from [3], we give in Theorem 3.6 the complete list of elements in $\mathcal{M}$. When $\operatorname{char}(\mathbf{k}) \neq 2$ and is good for $G$ (see Remark 3 for 
more detail), the list of elements in $\mathcal{M}$ coincides with that in [3. Corollary 4.2] for the elements $m_{C}$ for $C$ spherical, and one thus has

$$
\begin{aligned}
\mathcal{M} & =\left\{m_{C} \in W: C \text { is a spherical conjugacy class in } G\right\} \\
& =\left\{m_{C} \in W: C \text { is a conjugacy class in } G\right\} .
\end{aligned}
$$

As the last part of the paper, we consider in $₫ 4$ the case of $G=S L(n+1, \mathbf{k})$. For any conjugacy class $C$ in $S L(n+1, \mathbf{k})$ and any involution $w \in W \cong S_{n+1}$, we show in Theorem 4.2 that

$$
C \cap(B w B) \neq \emptyset \quad \text { iff } \quad l_{2}(w) \leq r(C)
$$

where $l_{2}(w)$ is the number of distinct 2-cycles in the cycle decomposition of $w$, and $r(C)=\min \{\operatorname{rank}(g-c I): c \in \mathbf{k}\}$ for any $g \in C$. Theorem 4.2 is proved in $4.4 \mathrm{using}$ a criterion of Ellers-Gordeev [11, Theorem 3.20]. The same criterion of Ellers-Gordeev also leads to a simple condition for a conjugacy class $\mathcal{O}$ in $W$ to lie entirely in $W_{C}$ for a given conjugacy class $C$ in $S L(n+1, \mathbf{k})$. See Theorem 4.8 .

A direct consequence of Theorem 4.2 is the following explicit description of the element $m_{C}$ for every conjugacy class $C$ of $S L(n+1, \mathbf{k})$ : for a positive integer $p$, let $\left[\frac{p}{2}\right]$ be the largest integer that is less than or equal to $\frac{p}{2}$. Let $m_{0}=1$, and for an integer $1 \leq l \leq\left[\frac{n+1}{2}\right]$, let $m_{l} \in S_{n+1}$ be the involution with the cycle decomposition

$$
m_{l}=(1, n+1)(2, n) \cdots(l, n+2-l) .
$$

Our Corollary 4.3 says that for any conjugacy class $C$ in $S L(n+1, \mathbf{k})$,

$$
m_{C}=m_{\left[\frac{n+1}{2}\right]} \quad \text { if } r(C) \geq\left[\frac{n+1}{2}\right], \quad \text { and } \quad m_{C}=m_{r(C)} \quad \text { if } r(C)<\left[\frac{n+1}{2}\right] .
$$

Note that $m_{\left[\frac{n+1}{2}\right]}$ is the maximal element in $S_{n+1}$. The explicit description of $m_{C}$ for an arbitrary conjugacy class $C$ in other classical groups will be given in [7].

Assume that $\operatorname{char}(\mathbf{k}) \neq 2$. Combining Theorem 4.2 and [3, Theorem 2.7], we show in Corollary 4.6 that $W_{C}=\left\{w \in S_{n+1}: w^{2}=1, l_{2}(w) \leq r(C)\right\}$ for a spherical conjugacy class $C$ in $S L(n+1, \mathbf{k})$.

1.2. Notation. Let $\Delta$ be the set of all roots of $G$ with respect to $H$, let $\Delta^{+} \subset \Delta$ be the set of positive roots determined by $B$, and let $\Gamma$ be the set of simple roots in $\Delta^{+}$. We also write $\alpha>0$ (resp. $\alpha<0)$ if $\alpha \in \Delta^{+}\left(\right.$resp. $\alpha \in-\Delta^{+}$).

For $\alpha \in \Gamma$, let $s_{\alpha} \in W$ be the reflection determined by $\alpha$. The length function on $W$ is denoted by $l$. For a subset $W_{1} \subset W$, an element $w \in W_{1}$ is called a minimal (resp. maximal) length element if $l(w) \leq l\left(w_{1}\right)$ (resp. $l(w) \geq l\left(w_{1}\right)$ ) for all $w_{1} \in W_{1}$. For a subset $J$ of $\Gamma$, let $W_{J}$ be the subgroup of $W$ generated by $\left\{s_{\alpha}: \alpha \in J\right\}$, let $w_{0, J}$ be the maximal length element in $W_{J}$, and let $W^{J} \subset W$ be the set of minimal length representatives of $W / W_{J}$. 
For $\delta \in \operatorname{Aut}(W)$, define the $\delta$-twisted conjugation of $W$ on itself by $u \cdot \delta v=\delta(u) v u^{-1}$ for $u, v \in W$, and for $w \in W$, let $\mathcal{O}_{w}^{\delta}=\left\{\delta(u) w u^{-1}: u \in W\right\}$ and call it the $\delta$-twisted conjugacy class of $w$ in $W$. Let $w_{0}=w_{0, \Gamma}$ be the longest element in $W$. We will use $\delta_{0}$ both for the permutation on $\Delta^{+}$given by $\delta_{0}(\alpha)=-w_{0}(\alpha)$ and for the automorphism of $W$ given by $\delta_{0}: W \longrightarrow W: \delta_{0}(w)=w_{0} w w_{0}$ for $w \in W$.

1.3. Acknowledgments. We thank X.-H. He and E. Ellers for helpful discussions and for drawing our attention to various references. We are especially grateful to G. Carnovale for answering our questions on spherical conjugacy classes and for her input to Remark 3. We thank the referees for helpful suggestions for the revised version of the paper. Our research was partially supported by HKRGC grants 703405 and 703407.

\section{The Sets $W_{C}$ AND $W_{C}^{-}$AND THE ELEMENTS $m_{C}$}

2.1. $W_{C}^{-}$in terms of $m_{C}$. Let the notation be as in $\$ 1.1$ and $\$ 1.2$. In particular, for each conjugacy class $C$ in $G$, the subsets $W_{C}$ and $W_{C}^{-}$of $W$ are given in (11) and (2).

Lemma 2.1. One has $W_{C} \subset W_{C}^{-}$for every conjugacy class $C$ in $G$.

Proof. Let $w \in W$. If $C \cap(B w B) \neq \emptyset$, then $C \cap(B w) \neq \emptyset$, so $C \cap\left(B w B^{-}\right) \neq \emptyset$.

\section{Q.E.D.}

Lemma 2.2. For any $w \in W$,

$$
B w B^{-} B=\bigsqcup_{w^{\prime} \in W, w \leq w^{\prime}} B w^{\prime} B .
$$

Proof. Clearly $B w B^{-} B$ is the union of some $(B, B)$-double cosets. Let $w^{\prime} \in W$. Then

$$
B w^{\prime} B \subset B w B^{-} B \Longleftrightarrow\left(B w^{\prime} B\right) \cap\left(B w B^{-} B\right) \neq \emptyset \Longleftrightarrow\left(B w^{\prime} B\right) \cap\left(B w B^{-}\right) \neq \emptyset,
$$

which, by [8, Corollary 1.2], is equivalent to $w \leq w^{\prime}$.

\section{Q.E.D.}

Lemma 2.3. Let $C$ be a conjugacy class in $G$ and let $w \in W$. Then $w \in W_{C}^{-}$if and only if $w \leq w^{\prime}$ for some $w^{\prime} \in W_{C}$.

Proof. Since $C$ is conjugation invariant,

$$
C \cap\left(B w B^{-}\right) \neq \emptyset \Longleftrightarrow C \cap\left(B w B^{-} B\right) \neq \emptyset,
$$

which, by Lemma 2.2, is equivalent to $w \leq w^{\prime}$ for some $w^{\prime} \in W_{C}$. 
Lemma 2.4. [2, §1] Let $C$ be a conjugacy class in $G$. Then

1) there is a unique $m_{C} \in W$ such that $C \cap\left(B m_{C} B\right)$ is dense in $C$;

2) $w \leq m_{C}$ for every $w \in W_{C}$.

Theorem 2.5. For every conjugacy class $C$ in $G, W_{C}^{-}=\left\{w \in W: w \leq m_{C}\right\}$.

Proof. Let $w \in W$. If $w \leq m_{C}$, then $w \in W_{C}^{-}$by Lemma 2.3. Conversely, if $w \in W_{C}^{-}$, then again by Lemma 2.3, $w \leq w^{\prime}$ for some $w^{\prime} \in W_{C}$. Since $w^{\prime} \leq m_{C}$ by Lemma 2.4, one has $w \leq m_{C}$.

\section{Q.E.D.}

Lemma 2.6. If $C$ and $C^{\prime}$ are two conjugacy classes in $G$ such that $C^{\prime} \subset \bar{C}$, then $m_{C^{\prime}} \leq m_{C}$.

Proof. Since $\emptyset \neq C^{\prime} \cap\left(B m_{C^{\prime}} B\right) \subset \bar{C}=\overline{C \cap\left(B m_{C} B\right)} \subset \overline{B m_{C} B}$, one has $m_{C^{\prime}} \leq m_{C}$.

\section{Q.E.D.}

A conjugacy class $C$ in $G$ is said to be central if $C=\{z\}$ for some $z$ in the center of $G$. It is clear that $m_{C}=1$ if $C$ is central.

Lemma 2.7. A conjugacy class $C$ in $G$ is central if and only if $m_{C}=1$.

Proof. Assume that $m_{C}=1$. Then $C \subset B$ by Lemma 2.4. Conjugating $C$ by a representative of $w_{0}$ in $N_{G}(H)$, one has $C \subset B^{-}$, so $C \subset H$. Let $z \in C$ be arbitrary. Let $\alpha$ be any root. If $z u z^{-1} \neq u$ for some $u \in U_{\alpha}$, the one-dimensional unipotent subgroup of $G$ defined by $\alpha$, then $u^{-1} z u=u^{-1} z u z^{-1} z \in U_{\alpha} z$ is not contained in $H$, contradicting the fact that $C \subset H$. Thus $z u z^{-1}=u$ for every $u \in U_{\alpha}$. Consequently $z$ is in the center of $G$ and $C=\{z\}$.

\section{Q.E.D.}

2.2. Some properties of $W_{C}$ and $W_{C}^{-}$. We recall some results from [10, 15].

Definition 1. 1) [10, Definition 3.1] Let $w, w^{\prime} \in W$. An ascent from $w$ to $w^{\prime}$ is a sequence $\left\{\alpha_{j}\right\}_{1 \leq j \leq k}$ in $\Gamma$ such that

$$
w^{\prime}=s_{\alpha_{k}} \cdots s_{\alpha_{2}} s_{\alpha_{1}} w s_{\alpha_{1}} s_{\alpha_{2}} \cdots s_{\alpha_{k}}
$$

and $l\left(s_{\alpha_{j}} \cdots s_{\alpha_{2}} s_{\alpha_{1}} w s_{\alpha_{1}} s_{\alpha_{2}} \cdots s_{\alpha_{j}}\right) \geq l\left(s_{\alpha_{j-1}} \cdots s_{\alpha_{2}} s_{\alpha_{1}} w s_{\alpha_{1}} s_{\alpha_{2}} \cdots s_{\alpha_{j-1}}\right)$ for every $1 \leq$ $j \leq k$. Write $w^{\prime} \longleftarrow w$ if there is an ascent from $w$ to $w^{\prime}$ or if $w^{\prime}=w$.

2) [15, §2.9] For $w, w^{\prime}, x \in W$, write $w \stackrel{x}{\sim} w^{\prime}$ if $l(w)=l\left(w^{\prime}\right), w^{\prime}=x w x^{-1}$, and either $l\left(w^{\prime}\right)=l(x w)+l(x)$ or $l\left(w^{\prime}\right)=l(x)+l\left(w x^{-1}\right)$. Write $w \sim w^{\prime}$ if there exist sequences of $\left\{x_{j}\right\}_{1 \leq j \leq k}$ and $\left\{w_{j}\right\}_{1 \leq j \leq k}$ in $W$ such that

$$
w \stackrel{x_{1}}{\sim} w_{1} \stackrel{x_{2}}{\sim} \ldots \stackrel{x_{k}}{\sim} w_{k}=w^{\prime}
$$


Proposition 2.8. [15, §2.9] Let $\mathcal{O}$ be any conjugacy class in $W$.

1) For any $w \in \mathcal{O}$, there exists a maximal length element $w^{\prime} \in \mathcal{O}$ such that $w^{\prime} \longleftarrow w$;

2) If $w^{\prime}$ and $w^{\prime \prime}$ are two maximal length elements in $\mathcal{O}$, then $w^{\prime} \sim w^{\prime \prime}$.

Proposition 2.9. Let $C$ be a conjugacy class in $G$, and let $w, w^{\prime} \in W$.

1) If $w^{\prime} \longleftarrow w$ and $w \in W_{C}$, then $w^{\prime} \in W_{C}$.

2) If $w \sim w^{\prime}$ and $w \in W_{C}$, then $w^{\prime} \in W_{C}$.

Proof. 1) is just [10, Proposition 3.4]. To see 2), assume that $w \stackrel{x}{\sim} w^{\prime}$ for some $x \in W$, so $w^{\prime}=x w x^{-1}$, and either $l\left(w^{\prime}\right)=l(x w)+l(x)$ or $l\left(w^{\prime}\right)=l(x)+l\left(w x^{-1}\right)$. Assume first that $l\left(w^{\prime}\right)=l(x w)+l(x)$. Then $B w^{\prime} B=B x w B x^{-1} B$, so

$$
C \cap\left(B w^{\prime} B\right)=C \cap\left(B x w B x^{-1} B\right) \supset C \cap\left(x w B x^{-1}\right) \neq \emptyset .
$$

Thus $C \cap\left(B w^{\prime} B\right) \neq \emptyset$, and so $w^{\prime} \in W_{C}$. The case of $l\left(w^{\prime}\right)=l(x)+l\left(w x^{-1}\right)$ is proved similarly.

\section{Q.E.D.}

Remark 1. We refer to [10, 11] for a more detailed study of the set $W_{C}$ and in particular for the case of $G=S L(n, \mathbf{k})$. It is proved in [4] by G. Carnovale that, when $\operatorname{char}(\mathbf{k}) \neq 2$ and is good for $G$, a conjugacy class $C$ in $G$ is spherical if and only if $W_{C}$ consists only of involutions.

Recall that for $w \in W, \mathcal{O}_{w}$ denotes the conjugacy class of $w$ in $W$.

Corollary 2.10. Let $C$ be any conjugacy class in $G$, and let $w \in W$. If $w \in W_{C}$, then $\mathcal{O}_{w} \subset W_{C}^{-}$.

Proof. Suppose that $w \in W_{C}$. By Proposition 2.8 and Proposition 2.9. every maximal length element in $\mathcal{O}_{w}$ is in $W_{C}$. Since maximal elements in $\mathcal{O}_{w}$ with respect to the Bruhat order are also maximal length elements [16, Corollary 4.5], Corollary 2.10 now follows from Theorem 2.5 .

\section{Q.E.D.}

\subsection{Some properties of $m_{C}$.}

Corollary 2.11. For every conjugacy class $C$ in $G, m_{C}$ is the unique maximal length element in $\mathcal{O}_{m_{C}}$.

Proof. By Proposition 2.8, there exists a maximal length element $w^{\prime} \in \mathcal{O}_{m_{C}}$ such that $w^{\prime} \longleftarrow m_{C}$. By Proposition 2.9, $w^{\prime} \in W_{C}$, so $w^{\prime} \leq m_{C}$ by Lemma 2.4. Since $l\left(w^{\prime}\right) \geq$ $l\left(m_{C}\right)$, one has $w^{\prime}=m_{C}$. Thus $m_{C}$ is a maximal length element in $\mathcal{O}_{m_{C}}$. If $w_{1}$ is any maximal length element in $\mathcal{O}_{m_{C}}$, then $w_{1} \in W_{C}$ by Proposition 2.8 and Proposition 2.9. 
and thus $w_{1} \leq m_{C}$ by Lemma 2.4. Since $l\left(w_{1}\right)=l\left(m_{C}\right)$, one has $w_{1}=m_{C}$. Thus $m_{C}$ is the only maximal length element in $\mathcal{O}_{m_{C}}$.

\section{Q.E.D.}

Corollary 2.12. For any conjugacy class $C$ in $G, w_{0} m_{C}$ is the unique minimal length element in the $\delta_{0}$-twisted conjugacy class $\mathcal{O}_{w_{0} m_{C}}^{\delta_{0}}$ (see notation in $\S 1.2$ ).

Proof. Corollary 2.12 follows from the fact that, for any $w \in W$, the bijection

$$
\phi: W \longrightarrow W: u \longmapsto w_{0} u, \quad u \in W,
$$

maps maximal length elements in $\mathcal{O}_{w}$ to minimal length elements in $\mathcal{O}_{w_{0}}^{\delta_{0}}$.

Q.E.D.

Remark 2. Let $\tilde{G}$ be the connected and simply connected cover of $G$ with covering map $\pi: \tilde{G} \rightarrow G$, and let $Z=\pi^{-1}(e)$, where $e$ is the identity element of $G$. Let $\tilde{A}=\pi^{-1}(A)$, where $A \in\left\{H, B, B^{-}\right\}$. Identify the Weyl group for $\tilde{G}$ with $W$. For any conjugacy class $C$ in $G, \pi^{-1}(C)$ is a union of conjugacy classes in $\tilde{G}$. Since $Z \subset \tilde{H}=\tilde{B} \cap \tilde{B}^{-}$, it is easy to see that for any conjugacy classes $\tilde{C}$ in $\pi^{-1}(C), W_{\tilde{C}}=W_{C}$ and $W_{\tilde{C}}^{-}=W_{C}^{-}$, and in particular, $m_{C}=m_{\tilde{C}}$. Thus the subset $\left\{m_{C}: C\right.$ a conjugacy class in $\left.G\right\}$ of $W$ depends only on the isogeny class of $G$.

2.4. $\delta$-twisted conjugacy classes. Let $\delta$ be an automorphism of $G$ such that $\delta(B)=$ $B$ and $\delta(H)=H$, and let $G$ act on itself by

$$
g \cdot{ }_{\delta} h=\delta(g) h g^{-1}, \quad g, h \in G .
$$

The $G$-orbits for this action will be called $\delta$-twisted conjugacy classes in $G$. In the study of a certain Poisson structure on the de Concini-Procesi compactification $\bar{G}$ of $G$ when $G$ is complex and of adjoint type [12], one needs to know when a $\delta$-twisted conjugacy class in $G$ intersects with a Bruhat cell $B w B^{-}$.

Given a $\delta$-twisted conjugacy class $C_{\delta}$ of $G$, let

$$
\begin{aligned}
& W_{C_{\delta}}=\left\{w \in W: C_{\delta} \cap(B w B) \neq \emptyset\right\}, \\
& W_{C_{\delta}}^{-}=\left\{w \in W: C_{\delta} \cap\left(B w B^{-}\right) \neq \emptyset\right\} .
\end{aligned}
$$

All the arguments in $\$ 2.1$ carry through when $C$ is replaced by $C_{\delta}$. In particular, $W_{C_{\delta}} \subset W_{C_{\delta}}^{-}$, and if $m_{C_{\delta}}$ denotes the unique element in $W$ such that $C_{\delta} \cap\left(B m_{C_{\delta}} B\right)$ is dense in $C_{\delta}$, then

$$
W_{C_{\delta}}^{-}=\left\{w \in W: w \leq m_{C_{\delta}}\right\} .
$$

Since $\delta(H)=H$ and $\delta(B)=B, \delta$ acts on the set $\Gamma$ of simple roots and on $W$ such that $\delta(w)=\delta \circ w \circ \delta^{-1}: \Delta \rightarrow \Delta$. Minimal length elements in $\delta$-twisted conjugacy classes in $W$ have been studied in [15, 16]. In particular, for $w, w^{\prime} \in W$, write $w^{\prime} \longleftarrow^{\delta} w$ 
if $w_{0} w \longrightarrow_{F} w_{0} w^{\prime}$ in the notation of [15, Definition $2.2(\mathrm{a})$ ], and write $w \sim^{\delta} w^{\prime}$ if $w_{0} w \sim_{F} w_{0} w^{\prime}$ in the notation of [15, Definition $\left.2.2(\mathrm{~b})\right]$, where $F=\delta^{-1} \delta_{0}$. Using the fact that the map $\phi$ in (6) maps maximal length elements in a $\delta$-twisted conjugacy class $\mathcal{O}_{w}^{\delta}$ to minimal length elements in the $\delta_{0} \delta$-twisted conjugacy class $\mathcal{O}_{w_{0} w}^{\delta_{0} \delta}$, one sees that [15, Theorem 2.6] is equivalent to the following generalization of Proposition 2.8:

Proposition 2.13. Let $\mathcal{O}^{\delta}$ be a $\delta$-twisted conjugacy class in $W$.

1) For any $w \in \mathcal{O}^{\delta}$, there exists a maximal length element $w^{\prime} \in \mathcal{O}^{\delta}$ such that $w^{\prime} \longleftarrow^{\delta}$ $w$;

2) If $w^{\prime}$ and $w^{\prime \prime}$ are two maximal length elements in $\mathcal{O}^{\delta}$, then $w^{\prime} \sim^{\delta} w^{\prime \prime}$.

Similarly, we have the following generalization of Proposition 2.9 .

Proposition 2.14. Let $C_{\delta}$ be a $\delta$-twisted conjugacy class in $G$, and let $w, w^{\prime} \in W$.

1) If $w^{\prime} \longleftarrow^{\delta} w$ and $w \in W_{C_{\delta}}$, then $w^{\prime} \in W_{C_{\delta}}$.

2) If $w \sim^{\delta} w^{\prime}$ and $w \in W_{C_{\delta}}$, then $w^{\prime} \in W_{C_{\delta}}$.

Proof. For 1), it is enough to assume that $w^{\prime}=s_{\delta(\alpha)} w s_{\alpha}$ for $\alpha \in \Gamma$ and $l\left(w^{\prime}\right) \geq l(w)$. Assume also that $w(\alpha) \neq \pm \delta(\alpha)$ because otherwise $w^{\prime}=w$ and we are done. Fix a representative $\dot{s}_{\alpha}$ of $s_{\alpha}$ in $N_{G}(H)$. If $w^{\prime}>w s_{\alpha}$, choosing $g \in C_{\delta} \cap B w$, one has

$$
\delta\left(\dot{s}_{\alpha}\right) g \dot{s}_{\alpha}^{-1} \in C_{\delta} \cap\left(B s_{\delta(\alpha)} B w s_{\alpha} B\right)=C_{\delta} \cap\left(B w^{\prime} B\right) .
$$

Assume that $w^{\prime}<w s_{\alpha}$. If $w^{\prime}<s_{\delta(\alpha)} w$, then $s_{\delta(\alpha)}(w(\alpha))<0$, and since $w(\alpha) \neq \pm \delta(\alpha)$, one has $w(\alpha)<0$, which implies that $w^{\prime}<w s_{\alpha}<w$, a contradiction. Hence $w^{\prime}>$ $s_{\delta(\alpha)} w$. Choose $g \in w B$. Then

$$
\delta\left(\dot{s}_{\alpha}\right) g \dot{s}_{\alpha}^{-1} \in C_{\delta} \cap\left(B s_{\delta(\alpha)} w B s_{\alpha} B\right)=C_{\delta} \cap\left(B w^{\prime} B\right) .
$$

For 2), assume, in the notation of [15, Definition $2.2(\mathrm{~b})$ ], that $w_{0} w \stackrel{x}{\sim} \delta^{-1} \delta_{0} w_{0} w^{\prime}$ for some $x \in W$. Then $w^{\prime}=\delta(y) w y^{-1}$, where $y=\delta^{-1} \delta_{0}\left(x^{-1}\right)$, such that either $l\left(w^{\prime}\right)=$ $l(\delta(y) w)+l(y)$ or $l\left(w^{\prime}\right)=l(y)+l\left(w y^{-1}\right)$. The rest of the proof of 2$)$ is similar to that of 2) of Proposition 2.9,

\section{Q.E.D.}

Corollary 2.15. For any $\delta$-twisted conjugacy class $C_{\delta}$ in $G, m_{C_{\delta}}$ is the unique maximal length element in its $\delta$-twisted conjugacy class in $W$.

Proof. Using Proposition 2.13 and Proposition 2.14, the proof of the statement is similar to that of Corollary 2.11 . 


\section{Conjugacy Classes of $W$ With unique maximal Length elements}

3.1. The set $\mathcal{M}$. By Corollary 2.11, $m_{C} \in \mathcal{M}$ for every conjugacy class $C$ in $G$, where $\mathcal{M}$ is the subset of $W$ given in (3). It is thus desirable to have a precise description of elements in $\mathcal{M}$. Clearly $\mathcal{M}$ is in one-to-one correspondence with conjugacy classes in $W$ that have unique maximal length elements.

It is easy to see that if $G=G_{1} \times G_{2} \times \cdots \times G_{k}$ is a product of simple groups $G_{j}$ with Weyl groups $W_{j}$ for $1 \leq j \leq k$, then

$$
\mathcal{M}=\mathcal{M}_{1} \times \mathcal{M}_{2} \times \cdots \times \mathcal{M}_{k}
$$

where for $1 \leq j \leq k, \mathcal{M}_{j} \subset W_{j}$ is defined as in (3). It is thus enough to determine $\mathcal{M}$ for $G$ simple. This will be done in 3.3 .

Lemma 3.1. If $m \in \mathcal{M}$, then $m^{2}=1$.

Proof. By [14, Corollary 3.2.14], $m^{-1} \in \mathcal{O}_{m}$. Since $l(m)=l\left(m^{-1}\right)$, one has $m=m^{-1}$.

\section{Q.E.D.}

\subsection{The correspondence between $\mathcal{M}^{\prime}$ and $\mathcal{J}^{\prime}$. Introduce}

$$
\mathcal{M}^{\prime}=\left\{m \in W: m^{2}=1 \text { and } m \text { is a maximal length element in } \mathcal{O}_{m}\right\} .
$$

By Lemma [3.1, $\mathcal{M} \subset \mathcal{M}^{\prime}$. We first determine $\mathcal{M}^{\prime}$.

It is well-known that elements in $\mathcal{M}^{\prime}$ correspond to special subsets of the set $\Gamma$ of simple roots. Indeed, minimal or maximal length elements in conjugacy classes of involutions in $W$ have been studied (see, for example, [14, 16, 18, 19, 20, 22] and especially [14, Remark 3.2.13] for minimal length elements, [19, Theorem 1.1] for maximal length elements, and [16, Lemma 3.6] for minimal length elements in twisted conjugacy classes). We summarize the results on $\mathcal{M}^{\prime}$ in the following Proposition [3.3, and we give a proof of Proposition 3.3 for completeness.

Definition 2. A subset $J$ of $\Gamma$ is said to have Property (1) if $J$ is $\delta_{0}$-invariant and $-w_{0}(\alpha)=-w_{0, J}(\alpha)$ for all $\alpha \in J$. Let $\mathcal{J}^{\prime}$ be the collection of all subsets $J$ of $\Gamma$ that have Property (1). For $J \in \mathcal{J}^{\prime}$, let $m_{J}=w_{0} w_{0, J}$. For $m \in \mathcal{M}^{\prime}$, let $J_{m}=\{\alpha \in \Gamma$ : $m(\alpha)=\alpha\} \subset \Gamma$.

Lemma 3.2. If $m \in \mathcal{M}^{\prime}$, then $m=w_{0} w_{0, J_{m}}$, and $J_{m} \in \mathcal{J}^{\prime}$.

Proof. By [23, Lemma 3.2], $s_{\alpha} m s_{\alpha}=m$ for any $\alpha \in \Gamma$ with $s_{\alpha} m>m$. By [23, Proposition 3.5], $m=w_{0} w_{0, J_{m}}$ and $J_{m} \in \mathcal{J}^{\prime}$. 
Proposition 3.3. 1) The map $\psi: \mathcal{M}^{\prime} \rightarrow \mathcal{J}^{\prime}: m \mapsto J_{m}$ is bijective with inverse given by $J \mapsto m_{J}$ for $J \in \mathcal{J}^{\prime}$.

2) For $J, K \in \mathcal{J}^{\prime}, m_{J}$ and $m_{K}$ are in the same conjugacy class in $W$ if and only if there exists $w \in W$ with $\delta_{0}(w)=w$ such that $w(J)=K$.

Proof. 1) Since $m=w_{0} w_{0, J_{m}}$ for every $m \in \mathcal{M}^{\prime}, \psi$ is injective. To show that $\psi$ is surjective, let $J \in \mathcal{J}^{\prime}$ and we will prove that $m_{J} \in \mathcal{M}^{\prime}$. Since $J$ is $\delta_{0}$-invariant, $m_{J}$ is an involution. Property (1) implies that $s_{\alpha} m_{J} s_{\alpha}=m_{J}$ for every $\alpha \in J$, so $w m_{J} w^{-1}=m_{J}$ for every $w \in W_{J}$. Thus, if $u=w m_{J} w^{-1}$ is an element in $\mathcal{O}_{m_{J}}$, we can assume that $w \in W^{J}$ (see notation in $\$ 1.2$ ). Then

$$
\begin{aligned}
l(u) & \leq l(w)+l\left(m_{J} w^{-1}\right)=l(w)+l\left(w_{0}\right)-l\left(w_{0, J} w^{-1}\right) \\
& =l(w)+l\left(w_{0}\right)-l\left(w_{0, J}\right)-l\left(w^{-1}\right)=l\left(m_{J}\right) .
\end{aligned}
$$

This shows that $m_{J}$ is of maximal length in $\mathcal{O}_{m_{J}}$, so $m_{J} \in \mathcal{M}^{\prime}$. To show that $\psi\left(m_{J}\right)=J$, note that $J \subset J_{m_{J}}=\left\{\alpha \in \Gamma: m_{J}(\alpha)=\alpha\right\}$. If $\alpha \in \Gamma \backslash J$, then $w_{0, J}(\alpha)>0$ so $m_{J}(\alpha)=w_{0} w_{0, J}(\alpha)<0$. It follows that $J_{m_{J}} \subset J$. Thus $J_{m_{J}}=J$, and $\psi\left(m_{J}\right)=J$. This shows that $\psi$ is surjective and that its inverse is given by $\psi^{-1}(J)=m_{J}$.

2) Assume that $J, K \in \mathcal{J}^{\prime}$ are such that $m_{J}$ and $m_{K}$ are conjugate in $W$. Since $w m_{J} w^{-1}=m_{J}$ for any $w \in W_{J}$, we may assume that $m_{K}=w m_{J} w^{-1}$ for some $w \in W^{J}$. Let $\alpha \in J$. It follows from $m_{K} w=w m_{J}$ and $w \in W^{J}$ that

$$
m_{K} w(\alpha)=w m_{J}(\alpha)=w(\alpha)>0 .
$$

Thus $w(\alpha) \in[K]^{+}$, where $[K]^{+}$denotes the set of positive roots in the linear span of $K$. Denote similarly by $[J]^{+}$the set of positive roots in the linear span of $J$. Then $w\left([J]^{+}\right) \subset[K]^{+}$. Since both $m_{J}$ and $m_{K}$ are maximal length elements in the same conjugacy class in $W, l\left(m_{J}\right)=l\left(m_{K}\right)$. Since

$$
l\left(m_{J}\right)=l\left(w_{0}\right)-\left|[J]^{+}\right| \quad \text { and } \quad l\left(m_{K}\right)=l\left(w_{0}\right)-\left|[K]^{+}\right|,
$$

one has $\left|[J]^{+}\right|=\left|[K]^{+}\right|$. Here for a set $A,|A|$ denotes the cardinality of $A$. Thus $w\left([J]^{+}\right)=[K]^{+}$. It follows that $w(J)=K$. Now $m_{K}=w m_{J} w^{-1}$ implies that $w_{0, K}=$ $\delta_{0}(w) w_{0, J} w^{-1}$, so $\delta_{0}(w)=w_{0, K} w w_{0, J}=w$.

Conversely, if $J, K \in \mathcal{J}^{\prime}$ are such that $w(J)=K$ for some $w \in W$ with $\delta_{0}(w)=w$, then $w_{0, K}=w w_{0, J} w^{-1}=\delta_{0}(w) w_{0, J} w^{-1}$, so $m_{K}=w m_{J} w^{-1}$.

\section{Q.E.D.}

3.3. The correspondence between $\mathcal{M}$ and $\mathcal{J}$. We now turn to the set $\mathcal{M}$. For a subset $J$ of $\Gamma$, an $\alpha \in J$ is said to be isolated if $\left\langle\alpha, \alpha^{\prime}\right\rangle=0$ for every $\alpha^{\prime} \in J \backslash\{\alpha\}$. The following Definition 3 is inspired by [3. Lemma 4.1]. 
Definition 3. A subset $J$ of $\Gamma$ is said to have Property (2) if for every isolated $\alpha \in J$, there is no $\beta \in \Gamma \backslash\{\alpha\}$ with the following properties:

a) $\langle\alpha, \alpha\rangle=\langle\beta, \beta\rangle$ and $\langle\beta, \alpha\rangle \neq 0$;

b) $\left\langle\beta, \alpha^{\prime}\right\rangle=0$ for all $\alpha^{\prime} \in J \backslash\{\alpha\}$;

c) $-w_{0}(\beta)=\beta$.

Lemma 3.4. If $m \in \mathcal{M}$, then $J_{m}$ has Properties (1) and (2).

Proof. Let $m \in \mathcal{M}$. By Lemma 3.1 and Lemma 3.2, $J_{m}$ has Property (1). Suppose that $\alpha \in J_{m}$ is an isolated point and that there exists $\beta \in \Gamma \backslash\{\alpha\}$ with properties a), b) and c) in Definition [3. Let $J_{m}^{\prime}=J_{m} \backslash\{\alpha\}$. Since $\alpha \in J_{m}$ is isolated, one has $w_{0}(\alpha)=-\alpha$, so $m=w_{0} s_{\alpha} w_{0, J_{m}^{\prime}}=s_{\alpha} w_{0} w_{0, J_{m}^{\prime}}$, and by b) and c), $m(\beta)=s_{\alpha} w_{0} w_{0, J_{m}^{\prime}}(\beta)=s_{\alpha} w_{0}(\beta)=$ $-s_{\alpha}(\beta)<0$. Thus $s_{\beta} m s_{\beta}=m s_{m(\beta)} s_{\beta}=m s_{\alpha} s_{\beta} s_{\alpha} s_{\beta}$. By a), $s_{\alpha} s_{\beta} s_{\alpha} s_{\beta}=s_{\beta} s_{\alpha}$, so $s_{\beta} m s_{\beta}=m s_{\beta} s_{\alpha}$, and thus $s_{\alpha} s_{\beta} m s_{\beta} s_{\alpha}=s_{\alpha} m s_{\beta}$. Since $l\left(s_{\alpha} m s_{\beta}\right) \geq l\left(s_{\alpha} m\right)-1=l(m)$, and since $m \in \mathcal{M}, s_{\alpha} m s_{\beta}=m$. It follows from $m s_{\alpha} m=s_{\alpha}$ that $s_{\alpha} s_{\beta}=1$ which is a contradiction.

Q.E.D.

Let $\mathcal{J}$ be the collection of all subsets $J$ of $\Gamma$ with Properties (1) and (2). A $J \in \mathcal{J}$ is said to be non-trivial if $J$ is neither empty nor the whole of $\Gamma$.

Identify $\Gamma$ with the Dynkin diagram of $G$ and a subset $J$ of $\Gamma$ with a sub-diagram of the Dynkin diagram. The following description of $\mathcal{J}$ for $G$ simple is obtained in [3, Corollary 4.2]. We include the list here for the convenience of the reader.

Lemma 3.5. Assume that $G$ is simple and of rank $n \geq 2$. The following is a complete list of non-trivial $J \in \mathcal{J}$ with points in $J$ painted black:

(1) $A_{n}: J_{l}=\left\{\alpha_{i}: l+1 \leq i \leq n-l\right\}$ for $1 \leq l \leq\left[\frac{n+1}{2}\right]-1$ :

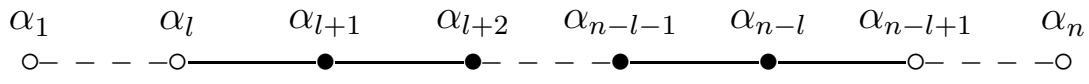

(2) $B_{n}: J_{1, l}=\left\{\alpha_{i}: l \leq i \leq n\right\}$ for $2 \leq l \leq n$ :

$$
\begin{aligned}
& \begin{array}{cccccc}
\alpha_{1} & \alpha_{l-1} & \alpha_{l} & \alpha_{l+1} & \alpha_{n-1} & \alpha_{n} \\
\circ--- & -0 & \bullet & \bullet
\end{array} \\
& J_{2, l}=\left\{\alpha_{1}, \alpha_{3}, \ldots, \alpha_{2 l-1}\right\} \cup\left\{\alpha_{i}: 2 l+1 \leq i \leq n\right\}, \text { for } 1 \leq l \leq \frac{n}{2}-1 \text { : } \\
& \begin{array}{cccccccc}
\alpha_{1} & \alpha_{2} & \alpha_{2 l-1} & \alpha_{2 l} & \alpha_{2 l+1} & \alpha_{2 l+2} & \alpha_{n-1} & \alpha_{n} \\
\bullet & 0- & ---\bullet & - & & \bullet & \bullet
\end{array} \\
& \text { If } n=2 m, J_{3}=\left\{\alpha_{1}, \alpha_{3}, \ldots, \alpha_{2 m-3}, \alpha_{2 m-1}\right\} \text { : } \\
& \begin{array}{cccccc}
\alpha_{1} & \alpha_{2} & \alpha_{2 m-3} & \alpha_{2 m-2} & \alpha_{2 m-1} & \alpha_{2 m} \\
\bullet & - & - & \longrightarrow
\end{array}
\end{aligned}
$$




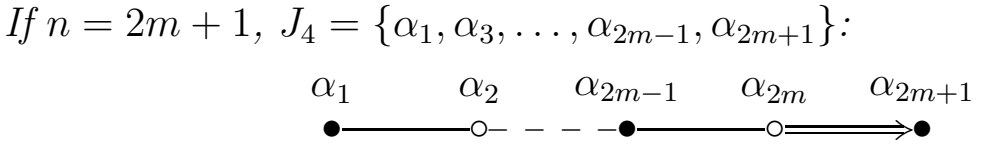

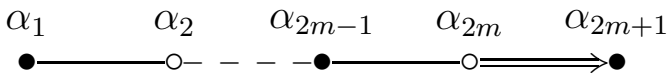

(3) $C_{n}: J_{1, l}=\left\{\alpha_{i}: l \leq i \leq n\right\}$ for $2 \leq l \leq n$ :

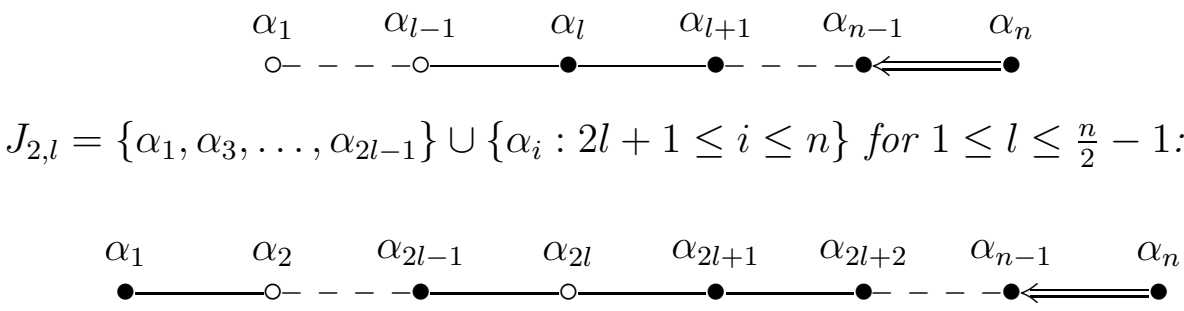

If $n=2 m, J_{3}=\left\{\alpha_{1}, \alpha_{3}, \ldots, \alpha_{2 m-3}, \alpha_{2 m-1}\right\}$ :

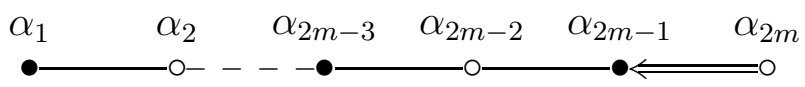

If $n=2 m+1, J_{4}=\left\{\alpha_{1}, \alpha_{3}, \ldots, \alpha_{2 m-1}, \alpha_{2 m+1}\right\}$ :

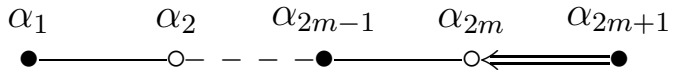

(4) $D_{2 m}: J_{1, l}=\left\{\alpha_{i}: 2 l-1 \leq i \leq 2 m\right\}$ for $2 \leq l \leq m$ :

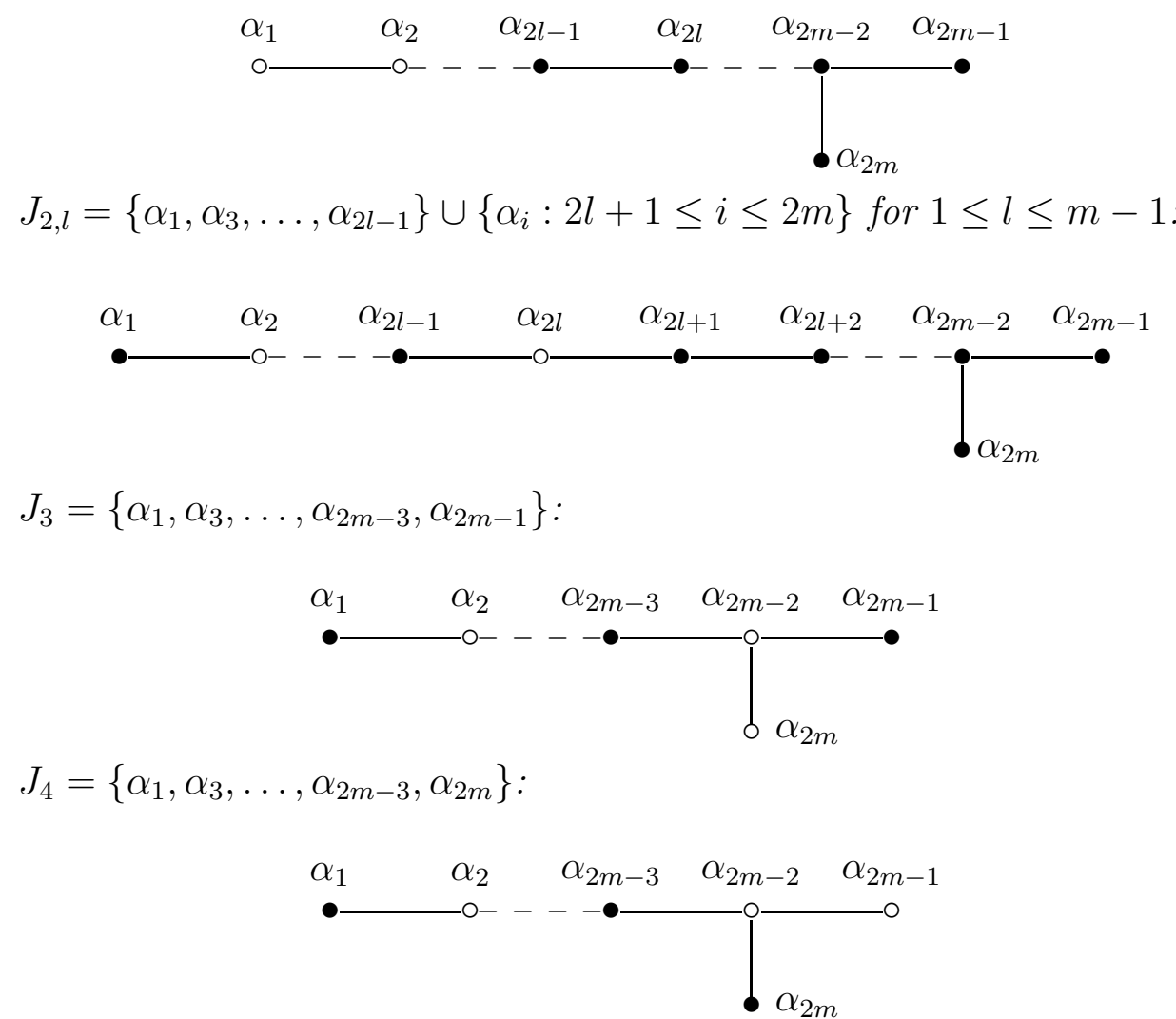


(5) $D_{2 m+1}: J_{1, l}=\left\{\alpha_{i}: 2 l-1 \leq i \leq 2 m+1\right\}$ for $2 \leq l \leq m$ :

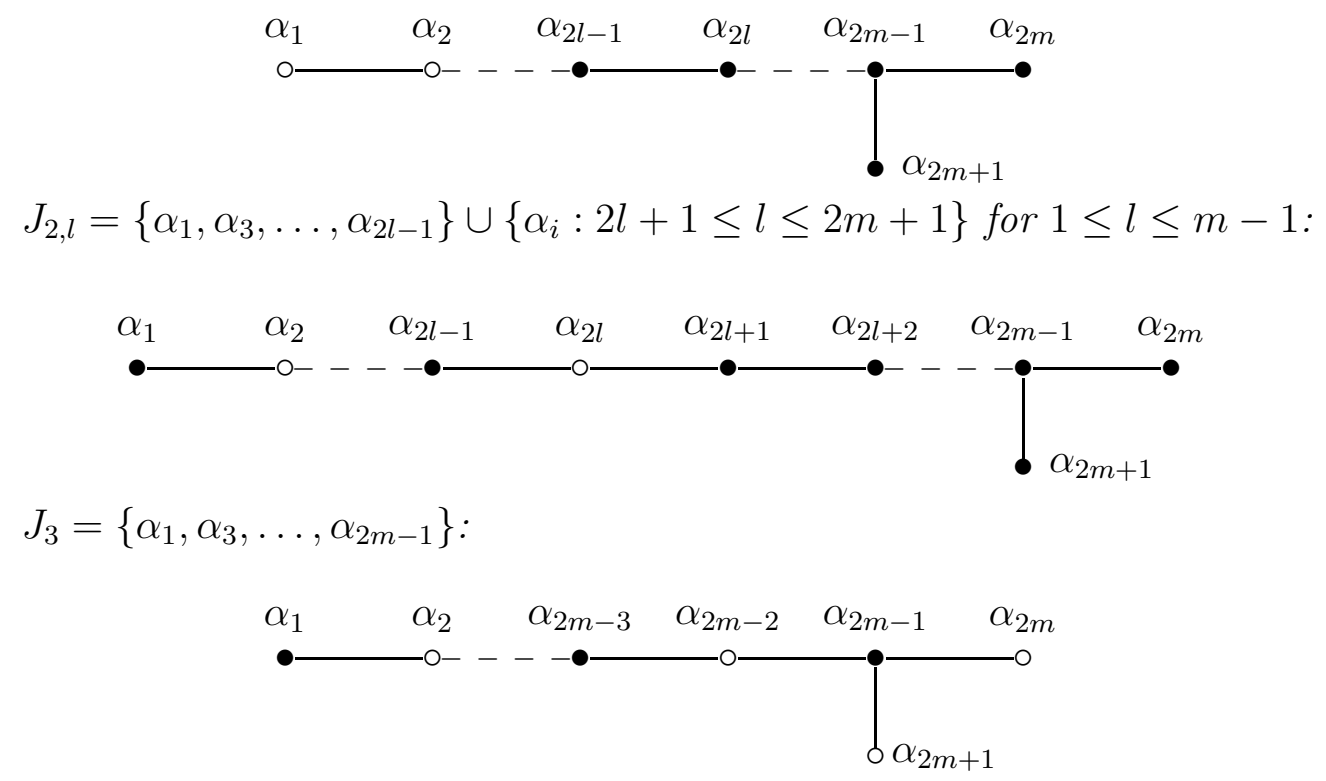

(6) $E_{6}$ :
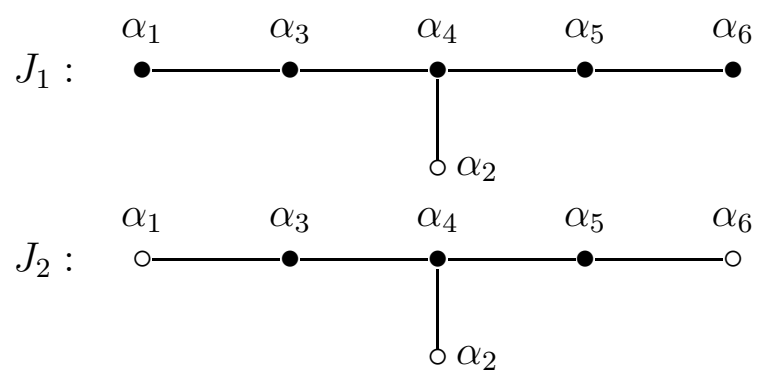

(7) $E_{7}$ :
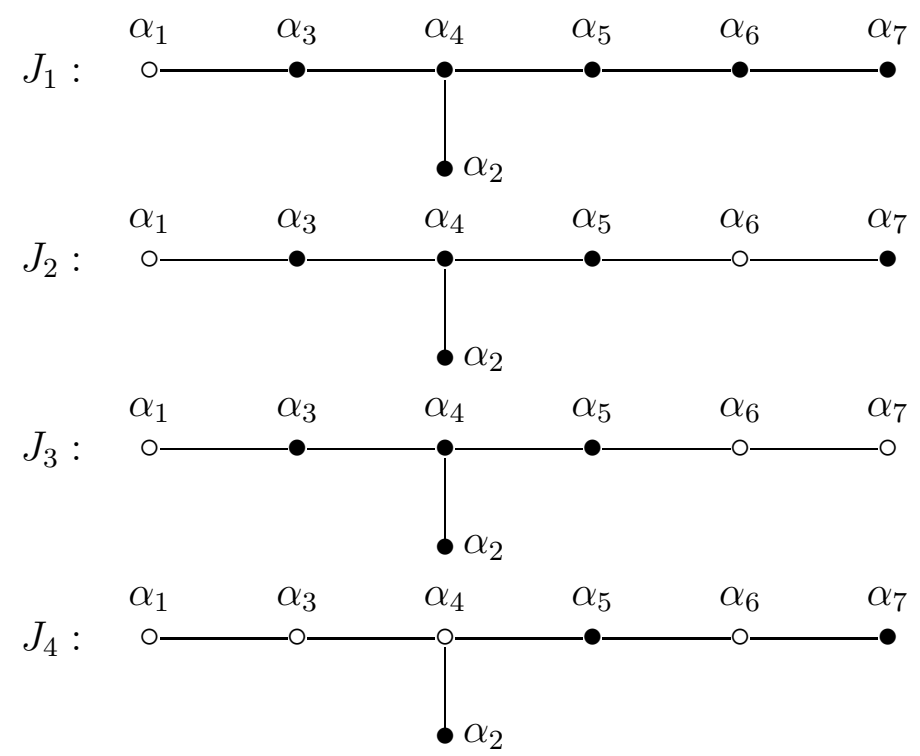
(8) $E_{8}$ :
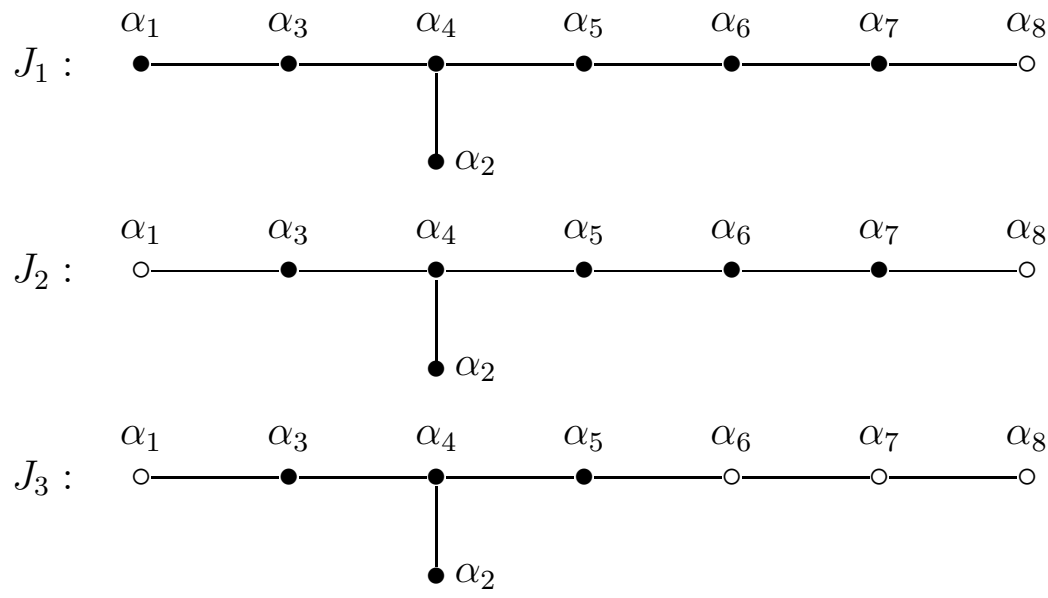

(9) $F_{4}$ :

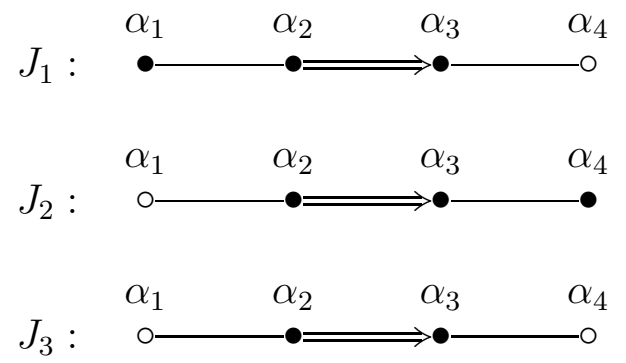

(10) $G_{2}$ :

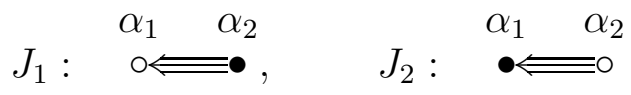

Theorem 3.6. When $G$ is simple, the map $\psi: \mathcal{M} \rightarrow \mathcal{J}: m \mapsto J_{m}$ is a bijection with inverse given by $\mathcal{J} \rightarrow \mathcal{M}: J \mapsto m_{J}$.

Proof. It is clear that $\psi$ is injective. To show that $\psi$ is surjective, let $J \in \mathcal{J}$. We need to show that $m_{J} \in \mathcal{M}$. Let $m$ be any maximal length element in $\mathcal{O}_{m_{J}}$. By Proposition 3.3, $m=m_{K}$, where $K \in \mathcal{J}^{\prime}$ and there exists $w \in W^{J}$ such that $w(J)=K$ and $\delta_{0}(w)=w$.

By examining the list of all non-trivial $J$ 's in $\mathcal{J}$ in Lemma 3.5 and the cases of $J=\emptyset$ or $J=\Gamma$, every $J \in \mathcal{J}$ uniquely embeds into the Dynkin diagram with Property (1) except in the cases of $J_{1, m}, J_{3}, J_{4}$ for $D_{2 m}$ and $J_{4}$ for $E_{7}$. In these cases, one can use results in [18] to check directly that $m_{J} \in \mathcal{M}$.

\section{Q.E.D.}

Remark 3. Let $G$ be simple. If $\mathbf{k}=\mathbb{C}$, then by [3, Remark 4.3], $m_{J}$ for every $J \in \mathcal{J}$ is equal to $m_{C}$ for some spherical conjugacy class $C$ in $G$. Consequently (44) and (5) hold. If $\mathbf{k}$ is algebraically closed with $\operatorname{char}(\mathbf{k}) \neq 2$ and good for $G$, spherical conjugacy classes in $G$ are studied in [3, 4] and classified in [5]. It was pointed out to us by G. Carnovale 
and by one of the referees that [3, Remark 4.3] is still valid when $\mathbb{C}$ is replaced by such a field k, so (4) and (5) still hold. It follows from $\$ 3.1$ that (4) and (5) also hold when $G$ is a product of simple groups.

Remark 4. When $G$ is simple, the minimal and maximal length elements as well as their numbers in conjugacy classes of involutions in $W$ are described in [18]. For the classical groups, the descriptions in [18] are in terms of (signed) 2-cycles for involutions in $W$ as opposed to sub-diagrams of the Dynkin diagram.

Remark 5. In [9], E. Ellers and N. Gordeev considered the intersections $C \cap\left(B^{-} w B\right)$, where $C$ is a non-central conjugacy class in a proper Chevalley group or finite twisted Chevalley group $G$ over an arbitrary field, and $w \in W$ is a product of simple reflections in which each simple reflection appears at most once. In particular, [9, Theorem 1] implies that such an intersection is always nonempty. In the notation of our paper, one has the following corollary of [9, Theorem 1], which can also be checked directly case by case.

Proposition 3.7. Assume that $G$ is simple. Then $w \leq m_{C}$ for every non-central conjugacy class $C$ in $G$ and every Coxeter element $w \in W$.

\section{The CASE OF $G=S L(n+1, \mathbf{k})$}

In this section, for an arbitrary conjugacy class $C$ in $S L(n+1, \mathbf{k})$, we give an explicit condition for $C \cap(B w B) \neq \emptyset$ when $w \in W \cong S_{n+1}$ is an involution. In particular, we describe $m_{C} \in S_{n+1}$ explicitly for every $C$.

4.1. Notation. As is standard, take the Borel subgroup $B$ (resp. $B^{-}$) to consist of all upper-triangular (resp. lower-triangular) matrices in $S L(n+1, \mathbf{k})$, so that $H=B \cap B^{-}$ consists of all diagonal matrices in $S L(n+1, \mathbf{k})$.

Identify the Weyl group $W$ of $S L(n+1, \mathbf{k})$ with the group $S_{n+1}$ of permutations on the set of integers between 1 and $n+1$. For $w \in S_{n+1}$, let

$$
l_{2}(w)=|\{i \in[1, n+1]: w(i)>i\}| .
$$

Every conjugacy class $C$ in $S L(n+1, \mathbf{k})$ contains some $g$ of (upper-triangular) Jordan form. We define the eigenvalues for $C$ to be the eigenvalues of such a $g \in C$ and similarly define the number and sizes of the Jordan blocks of $C$ corresponding to an eigenvalue. Let $I_{n+1} \in S L(n+1, \mathbf{k})$ be the identity matrix. For $g \in G L(n+1, \mathbf{k})$, define

$$
\begin{aligned}
& d(g)=\max \left\{\operatorname{dim} \operatorname{ker}\left(g-c I_{n+1}\right): c \in \mathbf{k}\right\} \\
& r(g)=n+1-d(g)=\min \left\{\operatorname{rank}\left(g-c I_{n+1}\right): c \in \mathbf{k}\right\} .
\end{aligned}
$$


For a conjugacy class $C$ in $S L(n+1, \mathbf{k})$, define $r(C)=r(g)$ for any $g \in C$, and let

$$
l(C)=\min \left\{r(C),\left[\frac{n+1}{2}\right]\right\} .
$$

\subsection{The main theorem and its consequences.}

Lemma 4.1. Let $C$ be a conjugacy class in $S L(n+1, \mathbf{k})$ and let $w \in S_{n+1}$. If $C \cap$ $(B w B) \neq \emptyset$, then $l_{2}(w) \leq r(C)$.

Proof. Assume that $C \cap(B w B) \neq \emptyset$. Let $g \in C \cap(B w B)$, and write $g=b_{1} \dot{w} b_{2}$, where $b_{1}, b_{2} \in B$ and $\dot{w}$ is any representative of $w$ in the normalizer of $H$ in $G$. Then for any non-zero $c \in \mathbf{k}$,

$$
\operatorname{rank}\left(g-c I_{n+1}\right)=\operatorname{rank}\left(b_{1} \dot{w} b_{2}-c I_{n+1}\right)=\operatorname{rank}\left(\dot{w}-c b_{1}^{-1} b_{2}^{-1}\right) .
$$

Let $l=l_{2}(w)$ and label the elements in the set $\{i \in[1, n+1]: w(i)>i\}$ as $i_{1}, \ldots, i_{l}$ such that $w\left(i_{1}\right)>\cdots>w\left(i_{l}\right)$. It is easy to see that for any $b \in B$, the columns of the matrix $\dot{w}-b$ corresponding to $i_{1}, \ldots, i_{l}$ are linearly independent. Indeed, for $1 \leq k \leq l$, let $v_{k}$ be the $i_{k}$ 'th column of $\dot{w}-b$. Suppose that $x_{1} v_{1}+\cdots+x_{l} v_{l}=0$, where $x_{k} \in \mathbf{k}$ for each $1 \leq k \leq l$. By looking at the $w\left(i_{1}\right)$ 'th coordinate of $x_{1} v_{1}+\cdots+x_{l} v_{l}$, one knows that $x_{1}=0$. Repeating this argument, one sees that $x_{k}=0$ for every $1 \leq k \leq l$. Consequently, $\operatorname{rank}(\dot{w}-b) \geq l=l_{2}(w)$ for every $b \in B$, and thus $\operatorname{rank}\left(g-c I_{n+1}\right) \geq l_{2}(w)$ for every non-zero $c \in \mathbf{k}$. It follows that $r(C)=r(g) \geq l_{2}(w)$.

\section{Q.E.D.}

Note that for an involution $w \in S_{n+1}, l_{2}(w)$ is the number of 2-cycles in the cycle decomposition of $w$, and $l_{2}(w) \leq\left[\frac{n+1}{2}\right]$.

Theorem 4.2. Let $C$ be a conjugacy class in $S L(n+1, \mathbf{k})$ and let $w \in S_{n+1}$ be an involution. Then $C \cap(B w B) \neq \emptyset$ if and only if $l_{2}(w) \leq l(C)$.

Theorem 4.2 will be proved in $\$ 4.4$ using a result of Ellers-Gordeev [11]. We have been informed by E. Ellers that in a yet to be published paper, E. Ellers and N. Gordeev have proved Theorem 4.2 for the case of $w=w_{0}$, the longest element in $S_{n+1}$, but for the groups $G L(n+1, \mathbf{k})$ and $S L(n+1, \mathbf{k})$ for an arbitrary field $\mathbf{k}$.

We now give some corollaries of Theorem 4.2. We first determine the element $m_{C}$ for every conjugacy class $C$ in $S L(n+1, \mathbf{k})$.

List the simple roots as $\Gamma=\left\{\alpha_{1}, \alpha_{2}, \ldots, \alpha_{n}\right\}$ in the standard way. Recall that $w_{0}$ is the longest element in $S_{n+1}$ and that for a subset $J$ of $\Gamma, w_{0, J}$ is the longest element in the subgroup of $S_{n+1}$ generated by simple roots in $J$. For an integer $0 \leq l \leq\left[\frac{n+1}{2}\right]$, let 
$m_{l}=w_{0} w_{0, J_{l}}$, where $J_{l}=\emptyset$ for $l=\left[\frac{n+1}{2}\right]$ and $J_{l}=\left\{\alpha_{l+1}, \ldots, \alpha_{n-l}\right\}$ for $0 \leq l \leq\left[\frac{n+1}{2}\right]-1$. Thus, $m_{0}=1$, and $m_{l}$ has the cycle decomposition

$$
m_{l}=(1, n+1)(2, n) \cdots(l, n+2-l), \quad \text { for } \quad 1 \leq l \leq\left[\frac{n+1}{2}\right] .
$$

In particular, $m_{l}=w_{0}$ for $l=\left[\frac{n+1}{2}\right]$. Note that for $0 \leq l_{1}, l_{2} \leq\left[\frac{n+1}{2}\right], m_{l_{1}} \leq m_{l_{2}}$ if and only if $l_{1} \leq l_{2}$.

Corollary 4.3. For any conjugacy class $C$ in $S L(n+1, \mathbf{k}), m_{C}=m_{l(C)}$, i.e., $m_{C}=w_{0}$ if $r(C) \geq\left[\frac{n+1}{2}\right]$ and $m_{C}=m_{r(C)}$ if $r(C)<\left[\frac{n+1}{2}\right]$.

Proof. Let $C$ be any conjugacy class in $S L(n+1, \mathbf{k})$. By Corollary 2.11, Lemma 3.4 and Lemma 3.5, $m_{C}=m_{l}$ for some $0 \leq l \leq\left[\frac{n+1}{2}\right]$. Since $C \cap\left(B m_{l} B\right) \neq \emptyset, l \leq l(C)$ by Theorem 4.2. Since $C \cap\left(B m_{l(C)} B\right) \neq \emptyset$ by Theorem 4.2, one also has $l(C) \leq l$. Thus $l=l(C)$.

Q.E.D.

Corollary 4.4. Let $C$ and $C^{\prime}$ be two conjugacy classes in $S L(n+1, \mathbf{k})$ such that $C^{\prime}$ is contained in the closure of $C$. Let $w \in S_{n+1}$ be an involution. If $w \in W_{C^{\prime}}$, then $w \in W_{C}$.

Proof. It follows from the definition that $r\left(C^{\prime}\right) \leq r(C)$, so $l\left(C^{\prime}\right) \leq l(C)$. Corollary 4.4 now follows directly from Theorem 4.2 .

\section{Q.E.D.}

Corollary 4.5. Let $\mathcal{O}$ be a conjugacy class of involutions in $S_{n+1}$ and let $C$ be a conjugacy class in $S L(n+1, \mathbf{k})$. If $\mathcal{O} \cap W_{C} \neq \emptyset$, then $\mathcal{O} \subset W_{C}$.

Proof. Since $l_{2}(w)=l_{2}\left(w^{\prime}\right)$ for every $w, w^{\prime} \in \mathcal{O}$, Corollary 4.5 follows directly from Theorem 4.2.

\section{Q.E.D.}

Consider spherical conjugacy classes in $S L(n+1, \mathbf{k})$. Assume that char $(\mathbf{k}) \neq 2$. By [5. Theorem 3.2], a spherical conjugacy class in $S L(n+1, \mathbf{k})$ is either semi-simple or unipotent. Moreover, a non-central semi-simple conjugacy class $C$ in $S L(n+1, \mathbf{k})$ is spherical if and only if it has exactly two distinct eigenvalues, and in this case, $r(C)$ is equal to the smaller multiplicity of the two eigenvalues. A unipotent conjugacy class $C$ in $S L(n+1, \mathbf{k})$ is spherical if and only if all of its Jordan blocks are of size at most 2 , and in this case, $r(C)$ is the number of size 2 Jordan blocks for $C$. In particular, $l(C)=r(C)$ for every spherical conjugacy class in $S L(n+1, \mathbf{k})$. 
Corollary 4.6. Let $G=S L(n+1, \mathbf{k})$ with $\operatorname{char}(\mathbf{k}) \neq 2$. Then $W_{C}=\left\{w \in S_{n+1}: w^{2}=\right.$ $\left.1, l_{2}(w) \leq r(C)\right\}$ for every spherical conjugacy class $C$ in $S L(n+1, \mathbf{k})$.

Proof. Let $C$ be a spherical conjugacy class in $S L(n+1, \mathbf{k})$. Since $\operatorname{char}(\mathbf{k}) \neq 2,[3$, Theorem 2.7] applies, and one knows that if $w \in W_{C}$, then $w$ is an involution, and by Theorem 4.2, $l_{2}(w) \leq r(C)$. Conversely, if $w \in S_{n+1}$ is an involution with $l_{2}(w) \leq r(C)$, then $w \in W_{C}$ by Theorem 4.2.

\section{Q.E.D.}

4.3. Partitions associated to conjugacy classes. Recall (see for example [11, Page 705]) that for an integer $p>0$, a partition of $p$ is a non-increasing sequence $\lambda=$ $\left(\lambda_{1}, \ldots, \lambda_{s}\right)$ of positive integers such that $\lambda_{1}+\cdots+\lambda_{s}=p$, and $s$ is called the length of $\lambda$. The shape of a partition $\lambda=\left(\lambda_{1}, \ldots, \lambda_{s}\right)$ of $p$ consists of $s$ rows of empty boxes left-aligned with $\lambda_{j}$ boxes on the $j$-th row for each $1 \leq j \leq s$. The partition $\lambda^{*}$ of $p$ whose shape is obtained from switching the rows and columns of the shape of $\lambda$ is called the dual of $\lambda$. Let $\lambda=\left(\lambda_{1}, \ldots, \lambda_{s}\right)$ and $\mu=\left(\mu_{1}, \ldots, \mu_{t}\right)$ be two partitions of $p$. Define $\lambda \leq \mu$ if $\sum_{j=1}^{k} \lambda_{j} \leq \sum_{j=1}^{k} \mu_{j}$ for every $1 \leq k \leq t$. One has (see [17, Section I.1.11]) $\lambda \leq \mu$ if and only if $\mu^{*} \leq \lambda^{*}$.

For integers $p>0$ and $0 \leq l \leq\left[\frac{p}{2}\right]$, let $\left(2^{l}, 1^{p-2 l}\right)=(2, \ldots, 2,1, \ldots, 1)$ be the partition of $p$ with 2 appearing exactly $l$ times.

Lemma 4.7. Let $p>0$ be an integer and let $0 \leq l \leq\left[\frac{p}{2}\right]$. Then for any partition $\mu=\left(\mu_{1}, \ldots, \mu_{s}\right)$ of $p,\left(2^{l}, 1^{p-2 l}\right) \leq \mu$ if and only if $s \leq p-l$.

Proof. One has $\left(2^{l}, 1^{p-2 l}\right) \leq \mu$ if and only if $\mu^{*} \leq\left(2^{l}, 1^{p-2 l}\right)^{*}$, and the latter is equivalent to $s \leq p-l$.

\section{Q.E.D.}

We now recall the partition $\tilde{\nu}^{*}(C)$ of $n+1$ associated to a conjugacy class $C$ in $S L(n+1, \mathbf{k})$ given in [11, Section 3.4]: let $c_{1}, \ldots, c_{k}$ be the distinct eigenvalues of $C$, and for $1 \leq j \leq k$, let $d_{j}>0$ and $n_{j, 1} \geq \cdots \geq n_{j, d_{j}}>0$ be respectively the number and the sizes of Jordan blocks with eigenvalue $c_{j}$. Arrange the list of the eigenvalues so that $d_{1} \geq \cdots \geq d_{k}$. Then $d_{1}=n+1-r(C)$. The partition $\tilde{\nu}^{*}(C)$ of $n+1$ is given by $\tilde{\nu}^{*}(C)=\left(\xi_{1}, \ldots, \xi_{d_{1}}\right)$, where for $1 \leq t \leq d_{1}, \xi_{t}=n_{1, t}+\cdots+n_{k, t}$ (if $d_{j}<d_{1}$ for some $2 \leq j \leq k$, we set $n_{j, s}=0$ for all $\left.d_{j}<s \leq d_{1}\right)$.

For $w \in S_{n+1}$, let $\lambda(w)$ be the partition of $n+1$ formed by the lengths of the cycles in the cycle decomposition of $w$.

We are now ready to prove Theorem 4.2 . 
4.4. Proof of Theorem 4.2. Let $C$ be a conjugacy class in $S L(n+1, \mathbf{k})$ and let $w \in S_{n+1}$ be an involution. If $C \cap(B w B) \neq \emptyset$, then $l_{2}(w) \leq r(C)$ by Lemma 4.1, so $l_{2}(w) \leq l(C)$. Conversely, assume that $l_{2}(w) \leq l(C)$, or, equivalently, $l_{2}(w) \leq r(C)$. We need to show that $C \cap(B w B) \neq \emptyset$. By [14, Theorem 3.2.9(a)], there exists a minimal length element $w^{\prime} \in \mathcal{O}_{w}$ and an ascent from $w^{\prime}$ to $w$. Thus, in the notation of [11], there is a tree $\Gamma_{w}$ with $w^{\prime} \in T\left(\Gamma_{w}\right)$. By [11, Theorem 3.20], it is enough to show that $\lambda\left(w^{\prime}\right) \leq \tilde{\nu}^{*}(C)$. Since $\lambda\left(w^{\prime}\right)=\left(2^{l_{2}(w)}, 1^{n+1-2 l_{2}(w)}\right)$, by Lemma 4.7, $\lambda\left(w^{\prime}\right) \leq \tilde{\nu}^{*}(C)$ if and only if $d_{1} \leq n+1-l_{2}(w)$ which is equivalent to $l_{2}(w) \leq r(C)$. This proves Theorem 4.2 .

4.5. A condition for $\mathcal{O} \subset W_{C}$. For a conjugacy class $\mathcal{O}$ in $S_{n+1}$, let $\lambda(\mathcal{O})=\lambda(w)$ for any $w \in \mathcal{O}$. Another consequence of [11, Theorem 3.20] is the following generalization of Corollary 4.5.

Theorem 4.8. Let $\mathcal{O}$ be a conjugacy class in $S_{n+1}$ and $C$ a conjugacy class in $S L(n+$ $1, \mathbf{k})$. Then $\mathcal{O} \subset W_{C}$ if and only if $\lambda(\mathcal{O}) \leq \tilde{\nu}^{*}(C)$.

Proof. By Proposition 2.8 and Proposition 2.9, $\mathcal{O} \subset W_{C}$ if and only if all minimal length elements of $\mathcal{O}$ are in $W_{C}$. If $w \in \mathcal{O}$ is a minimal length element, let $\Gamma_{w}$ be the tree consisting only of $w$. By [11, Theorem 3.20], $w \in W_{C}$ if and only if $\lambda(\mathcal{O}) \leq \tilde{\nu}^{*}(C)$.

\section{Q.E.D.}

\section{REFERENCES}

[1] N. Cantarini, Spherical orbits and quantized enveloping algebras, Comm. Alg. 27 (7) (1999), $3439-3458$.

[2] N. Cantarini, G. Carnovale, and M. Costantini, Spherical orbits and representations of $\mathcal{U}_{\epsilon}(\mathfrak{g})$, Trans. Groups 10 (1) (2005), 29 - 62.

[3] G. Carnovale, Spherical conjugacy classes and involutions in the Weyl group, Math. Z. 260 (1) (2008), 1 - 23.

[4] G. Carnovale, Spherical conjugacy classes and the Bruhat decomposition, Ann. Inst. Fourier (Grenoble) 59(6) (2009), 2329-2357.

[5] G. Carnovale, A classification of spherical conjugacy classes in good characteristic, to appear in Pac. J. Math..

[6] M. Costantini, On the coordinate ring of spherical conjugacy classes, Math. Z. 264 (2010), $327-359$.

[7] K. Y. Chan, MPhil thesis in Mathematics, The University of Hong Kong, 2010.

[8] V. Deodhar, On some geometric aspects of Bruhat orderings, I. A finer decomposition of Bruhat cells, Invent. Math. 79 (1985), 499 - 511.

[9] E. Ellers and N. Gordeev, Intersections of conjugacy classes of Chevalley groups with Gauss cells, J. Alg. 220 (1999), 591 - 611.

[10] E. Ellers and N. Gordeev, Intersections of conjugacy classes with Bruhat cells in Chevalley groups, Pac. J. Math. 214 (2) (2004), 245 - 261.

[11] E. Ellers and N. Gordeev, Intersections of conjugacy classes with Bruhat cells in Chevalley groups: the cases of $S L_{n}(K), G L_{n}(K)$, J. P. and App. Alg. 209 (2007), 703 - 723.

[12] S. Evens and J.-H. Lu, On the variety of Lagrangian subalgebras, II, Ann. Sci. École Norm. Sup. 39 (2) (2006), 347 - 379. 
[13] S. Evens and J.-H. Lu, Poisson geometry of the Grothendieck resolution of a complex semisimple group, Moscow Math. J. 7 (4) (special volume in honor of V. Ginzburg's 50'th birthday) (2007), 613 - 642.

[14] M. Geck and G. Pfeiffer, Characters of finite Coxeter groups and Iwahori-Hecke algebras, London Mathematical Society Monographs, New Series, 21, Oxford University Press, 2000

[15] M. Geck, S. Kim, and G. Pfeiffer, Minimal length elements in twisted conjugacy classes of finite Coxeter groups, J. Alg. 229 (2) (2000), 570 - 600.

[16] X.-H. He, Minimal length elements in some double cosets of Coxeter groups, Adv. Math. 215 (2007), $469-503$.

[17] I. G. Macdonald, Symmetric functions and Hall polynomials, second edition, Oxford University Press, 1995.

[18] S. Perkins and P. Rowley, Minimal and maximal length involutions in finite Coxeter groups, Comm. Alg. 30 (3) (2002), 1273 - 1292.

[19] S. Perkins and P. Rowley, Lengths of involutions in Coxeter groups, J. Lie Theo. 14 (2004), $69-71$.

[20] R. W. Richardson, Conjugacy classes of involutions in Coxeter groups, Bull. Aust. Math. Soc. 26 (1982), 1 - 15.

[21] R. W. Richardson, Intersections of double cosets in algebraic groups, Indagationes Mathematicae 3 (1) (1992), 69-77.

[22] T. A. Springer, Some remarks on involutions in Coxeter groups, Comm. Alg. 10 (1982), 631 -633 .

[23] T. A. Springer, Some results on algebraic groups with involutions, Algebraic groups and related topics, Adv. Studies in Pure Math. 6 (1985) 525 - 543.

Department of Mathematics, Hong Kong University, Pokfulam Rd., Hong Kong

E-mail address:keiyuen@graduate.hku.hk, jhlu@maths.hku.hk, h0389481@graduate.hku.hk 